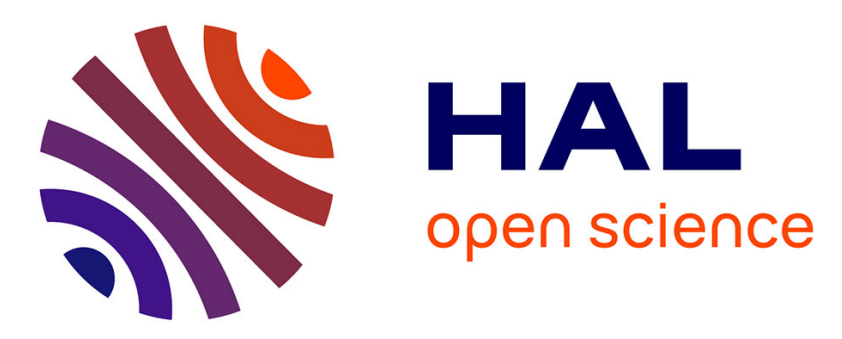

\title{
Multi-scale Study of Desiccation Shrinkage in Granular Soils
}

\author{
H. Bista, Liang Bo Hu, Boleslaw Mielniczuk, Moulay Saïd El Youssoufi, \\ Lyesse Laloui, Tomasz Hueckel
}

\section{- To cite this version:}

H. Bista, Liang Bo Hu, Boleslaw Mielniczuk, Moulay Saïd El Youssoufi, Lyesse Laloui, et al.. Multiscale Study of Desiccation Shrinkage in Granular Soils. UNSAT2014, Unsaturated Soils: Research \& Applications, Jul 2014, Sydney, Australia. pp.883-890. hal-01103269

\section{HAL Id: hal-01103269 \\ https://hal.science/hal-01103269}

Submitted on 14 Jan 2015

HAL is a multi-disciplinary open access archive for the deposit and dissemination of scientific research documents, whether they are published or not. The documents may come from teaching and research institutions in France or abroad, or from public or private research centers.
L'archive ouverte pluridisciplinaire HAL, est destinée au dépôt et à la diffusion de documents scientifiques de niveau recherche, publiés ou non, émanant des établissements d'enseignement et de recherche français ou étrangers, des laboratoires publics ou privés. 


\title{
Multi-scale study of desiccation shrinkage in granular soils
}

\author{
H. Bista \& L.B. Hu \\ Department of Civil Engineering, University of Toledo, Toledo, $\mathrm{OH}, \mathrm{USA}$ \\ B. Mielniczuk \\ Institut de Radioprotection et de Sâreté Nucléaire, IRSN, B.P. 3, Saint-Paud-lez-Durance Cedex, France. \\ Laboratoire de Micromécaniqué et d'Intégrité des Structures, MIST Laboratory, IRSN-CNRS-Université de \\ Montpellier 2, France
}

M.S. El Youssoufi

LMGC UMR UM2-CNRS 5508, Université Montpellier 2, Montpellier Cedex 5, France

Laboratoire de Micromécanique et d'Intégrité des Structures, MIST Laboratory. IRSN-CNRS-Universite de Montpellier 2, France

L. Laloui

Laboratory of Soil Mechanics, EPFL, Lausanne, Switzerland

T. Hueckel

Department of Civil and Environmental Engineering, Duke University, Durham, NC, USA

\begin{abstract}
This paper aims to identify and evaluate various eritical mechanisms associated with the processes of desiccation shrinkage in drying silty soils. A previously developed ID bundle-of-tubes model is refined to simulate the various stages of drying shrinkage in 2D using the actual pore size distribution based on Mercury Intrusion Porosimetry (MIP) data. It is revealed that the resulting shrinkage evolution is affected by air entry that may occur in two possible scenarios: air incursion at the external surface and formation of vapor nucleus in the interior. The analysis of mechanical deformation is coupled with the numerical simulation of the drying process which can be often characterized as a two-stage development, consisting of a constant rate period and a falling rate period. Numerical simulation of the drying rate evolution suggests that it may be closely associated with the onset of air entry and/or the progress of desaturation. Further transition of solid-water structural configuration into funicular and pendular states from initially capillary state is simulated.
\end{abstract}

\section{INTRODUCTION}

Desiccation is the continuous process of pore water loss from a soil exposed to drier or warmer environment. Evaporation of soil moisture results in moisture gradients and generates shrinkage of the material. When shrinkage is restrained, stress is generated, cracks would likely occur if tensile strength is exceeded. However, the underlying mechanisms of these processes are very complicated, involving sequential or even coupled scenarios of moisture evaporation and transport, drying shrinkage and shrinkage limit, air entry and onset of desaturation, and eventually formation and then evolution and rupture of liquid bridges, or if constrained, generation of concentrated tensile stress at the tip of flaws.

The present study aims to identify and quantitatively evaluate various critical mechanisms associated with the processes of desiccation shrinkage in multiple scales. There are two strong arguments for a multi-physics, multi-scale approach: the shrinkage deformation resulting from drying is under strong influence of capillary phenomena that must be addressed at a microscopic level; and the eventual cracking may be attributed to imperfections resulting from pore water removal and depletion in the evolving solid-pore structure at the meso-scale.

The analysis of mechanical deformation is coupled with the numerical simulation of the drying process which can be often characterized as a two-stage development, consisting of a constant rate period and a falling rate period. Of particular interest are the scenarios of air entry and the subsequent evolution of different stages of saturation consisting of funicular and pendular stages, conceptually understood as characterized by a 
continuous liquid phase and a continuous gas phase, respectively, Quantitative description or simulation of funicular and pendular states of water desaturation has been elusive, except some studies focused on isolated two- or three-sphere configurations (e.g. Urso et al. 1999). Lack of quantitative description of transition of these different states likely results from an inadequate understanding of a multi-scale approach to bring together experimental possibilities and findings, conceptual hypotheses and numerical models and devices. The presented work is an attempt to develop such an approach to provide a basis for a realistic scenario to model the complex set of phenomena.

\section{EXPERIMENTAL BACKGROUND}

Considerations can be made at three seales for desiccation drying: macro-scale continuum, at which stress, effective stress and strength criteria are formulated and soil experiments are conducted; meso-scale of clusters of grains and pores, where structural of air entry effects can be experimentally observed and analyzed; and micro-scale of single pore or grain water/vapor, where capillary forces can be observed. In this context Hu et al. (2013a, 2013b) examined microscopic data of the pore system evolution as represented by the mereury porosimetry results and postulated eorresponding mechanisms based on the pressure (suction) development in the pore-vessels and ensuing water cavitation at a critical suction. The event of air entry plays a central role in the process of desiccation cracking; it is envisioned that it may signify the onset of desaturation stage. Shrinkage stops practically simultaneously with the air entrance into the soil, at the water content value (hence referred to as shrinkage limit) still above $20 \%$ for the tested soils (Peron et al. 2009). The remaining drying process occurs with a much reduced shrinkage rate, but almost entirely via desaturation (e.g. Fredlund \& Rahardjo 1993, Kodikara et al. 1999, Pellenq et al. 2009).

The air entry phenomenon, despite that it occurs at the scale of a single pore, is considered a macroscopic behavior of soil, mainly based on the assumption of the equality of the fluid pressure at both scales. A suggestive air entry mechanism in a 2D granular system has been proposed by Childs (1969) shown in terms of a succession of stages during drying and re-wetting (Fig. Ia).

However, recent experiments at a meso-scale with drying of model clusters of smooth glass grains (Mielniczuk et al. 2013) with the funicular water bound between them reveal that there are more than a single air entry mode. The most characteristic two modesidentified in experimental

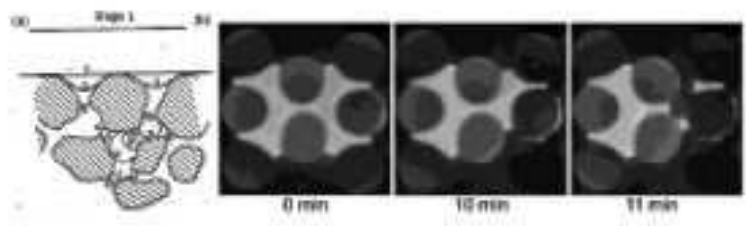

Figure I. (a) Succession of meniscus evolution during suction dewatering and rewetting, proposed by Childs (1969). (b) Evolution of the water body between 8 glass spheres subjected to evaporation at constant temperature, constant ambient vapor pressure, $\mathrm{A}$ localized nonsymmetric unstable mode of the interface evolution (air entry finger) is observed.

systems tested of $3,4,5,6$ and 8 silica grains in distilled water were two classes corresponding to a negative or positive local Gaussian curvature (which is a product of the two principal curvatures) of the surface of the local meniscus. Most typically, for a negative Gaussian curvature (or concave/convex meniscus) a non-symmetric mode of the unstable evolution of the water body constitutes a most common air entry mechanism into the body of the saturated soil. While the circumstances and numerical criterion for the event are still an open question, it clearly is an unstable and localized displacement of the gas/liquid inter-face in form of a finger, occurring at a several orders of magnitude higher rate than the preceding process manifested among others by a slow evolution of the liquid/gas interface.

Figure $1 \mathrm{~b}$ shows three of a series of images of a 2D saturated cluster of 8 grains (Mielniczuk et al. 2013) shot every 90 seconds during natural isothermal drying (using Canon EOS 500D camera). Grains were $3.5 \mathrm{~mm}$ in diameter ultra fine silica (glass) spheres, while the liquid is ultra-pure deionized water. As indicated, it took 10 minutes from the initial configuration to reach the intermediate one, and less than 90 additional seconds to develop the final configuration with the gas penetration. The depth of the penetration of the gas finger is about 4 times the average size (diameter) of the pore.

An alternative, symmetric entry mode takes place at a point with a positive Gaussian curvature (concave/concave meniscus) between the spheres and consists of a multi-step process: starting with a convergence of two gas/liquid interfaces situated oppositeone another (at the front and back of the picture), followed by formation of a suspended thin film of liquid, followed by a further thinning of the film, leading to coalescence of the two surfaces, undergoing what seems like a 2-D water cavitation, which subsequently propagates symmetrically with a circular projection until its boundary reaches the solid walls of the sphere. This is shown in Figure 2 for a six-sphere cluster. Notably, the 


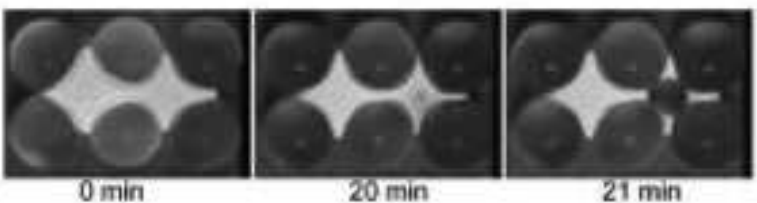

Figure 2. Evolution of the water body between 6 glass spheres subjected to evaporation at constant temperature, constant ambient vapor pressure. A symmetric localized unstable mode of the interface evolution is observed.

non-symmetrical scenario occurs for systems with higher separations between grains, while for lower separations a symmetrical seenario takes place. The unstable part of the process starts at the point when the thin film of water bifurcates. The bifurcations of thin films and thin sheets have been known for some time (c.g. Taylor 1959), but for capillary bridges have been observed only recently by Maeda et al. (2003) with a mixture of water and vapor molecules, of an intermediate density.

\section{SCENARIOS OF EVOLUTION OF THE DRYING STAGES}

\subsection{Macro-scale simulation of drying evolution}

A macro-scale simulation is performed against experimental results to illustrate a two-stage development of drying rates. As shown in Peron et al. (2005) and numerous resources, soil drying begins with an almost constant drying rate for a significant period (termed CRP, constant rate period), followed by a period of a sharply reduced drying rate (termed FRP, falling rate period). Similar process in non-deformable materials had been studied as early as back in $1920 \mathrm{~s}$ (e.g. Sherwood $1929)$ with analytical models being proposed. Puyate \& Lawrence (2006) developed simplified models with particular interest in cases at moderate drying intensity. Figure 3 shows the experimental results of drying rate observed in Peron et al. (2005) and the corresponding modelling results. A constant rate of $5.1 \times 10^{-7} \mathrm{~kg} / \mathrm{s}$ of water loss was measured in the experiment of a silty soil (Bioley silt) for the first $2000 \mathrm{hrs}$ and then the rate dropped dramatically.

The above mentioned models were established for a diffusion driven process, thus may not be aimed at or even applicable to a deformable medium which responds to desiceation drying with shrinkage deformation and the pore remains saturated for a long period of time. The declining drying rate may be associated with or influenced by the onset of air entry and/or the progress of desaturation. Such hypotheses of possible scenarios may be evaluated through modeling of phenomena occurring

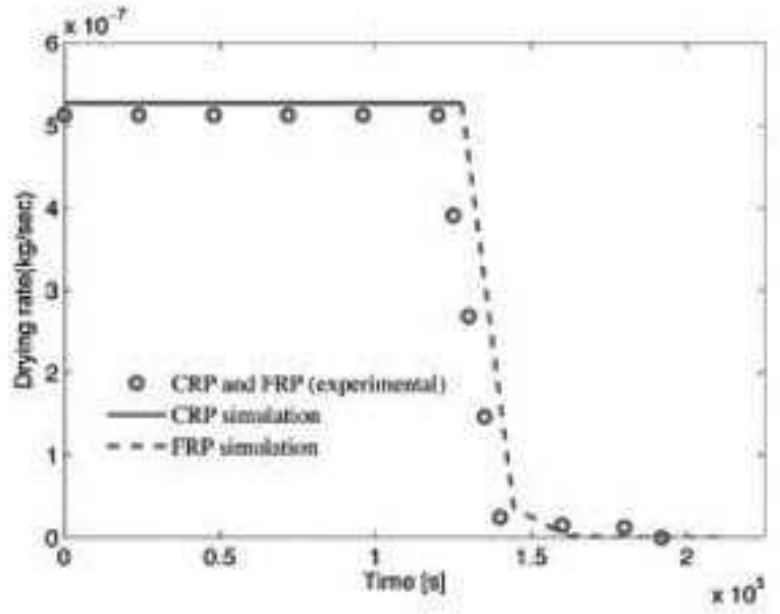

Figure 3. Constant drying rate period and falling rate period, experimental (Peron et al. 2005) and simulation results.

at the micro or meso scale. Hu et al. (2013a, 2013b) developed a meso-scale tubular model to simulate ID shrinkage of drying silty soils, based on the Mercury Intrusion Porosimetry (MIP) results of evolution of the pore size distribution. Despite its geometric simplicity, it yields reasonable agreement with experimental observation of shrinkage characteristics of drying soils with different pore liquids (Hu et al. 2013c). However, due to its ID configuration, it is incapable of addressing the transition of funicular to pendular stage of liquid saturation. The presented study extends this model to a $2 \mathrm{D}$ setting, allowing one to numerically identify and evaluate the evolution of capillary, funicular and pendular stages. In particular, the first mode of air entry presented in the preceding section can be readily simulated in the previous model. Hence, we are specifically interested in the second mode of air entry.

\subsection{Micro-scale simulation of drying evolution}

\subsubsection{Conceptual model}

The modeling started with the observation that the system of pores detected via mereury porosimetry evolves in a particular way during drying $\left(\mathrm{c}_{\mathrm{g}} \mathrm{g}_{\mathrm{i}}\right.$ in Bioley silt, Peron et al. 2005; Peron et al. 2009). At different stages of drying, i.e. at the onset at liquid limit, LL, then near shrinkage limit, SL, and at the nearly complete desaturation, two classes of pores are seen to evolve in a distinetly different way. These classes, referred to as Large Pores (LP) of around $1.5 \mu \mathrm{m}$ in diameter and Small Pores (SP) of $0.5 \mu \mathrm{m}$ do not form any particularly distinet modes originally, but their evolution makes them distinct.

To reproduce this evolution, the medium is idealized as consisting of a bundle of parallel cylindrical deformable vessels with two initial diameters, 
corresponding to LP and SP. While we discuss only 1-D shrinkage, in a more general case the elementary volumes, the pore-vessels can be arranged as in Figure 4 with 2-D intercalating layers of tubes oriented orthogonally ones to the others. As a result all quantities are appropriately weighed averages of the values pertaining to individual tubes. The vapor flux applied at the pore-vessel end imposes the Poiseuille flow of water throughout the vessel, associated with the shrinkage of tubes, commensurate with the volume of water removed. Hu et al. (2013a, 2013b) established the formulation of viscous flow through a deformable single (straight) microscopic vessel in terms of the fluid pressure in the pore-vessel, $p(x, t)$, based on Fung's equation for pressure vessel deformation (Fung 1984). This model is modified with a conventional linear elastic pressure-displacement formulation (e.g., Hill 1950; Chen \& Han 1988). The motivation for this modification is the possibility of the available analytical solution, allowing future consideration of a wide range of pore classes (even it is not explored in the present analysis), because now the formulation is a parabolic Partial Differential Equation (PDE).

$$
\frac{\partial p}{\partial t}=\lambda \frac{\partial^{2} p}{\partial x^{2}}, \quad \lambda=\frac{a^{2} E\left(b^{2}-a^{2}\right)}{16 \mu\left[a^{2}(1-2 \nu)+b^{2}(1+v)\right]}
$$

where $E$ denotes the stiffness of solid, $v$ is the Poisson's ratio, $\mu$ is the viscosity of pore fluid, $a, b$ are the initial internal and external radius of pore respectively. The solution is obtained analytically using the initial condition as $p=p_{0}=0$ at $t=0$ and the boundary conditions are: at $x=0, \partial p / \partial x=0$ and at $x=L_{,} D \partial p / \partial x=F$.

$$
p(x, t)=\frac{F L}{D}\left[r+\frac{2}{\pi^{2}} \sum_{n=1}^{\infty}\left(\begin{array}{l}
\frac{\cos (n \pi x)}{n^{2}} \\
-\frac{\cos (n \pi x)}{n^{2}} e^{-(n \pi)^{2} r}
\end{array}\right)\right]
$$

where $F$ is the evaporation flux and $D=-a^{2} / 8 \mu$, two dimensionlessvariablesare used, $X=(L-x) / L$ and $r=\lambda t / L^{2}$.

The above formulation and considerations are valid only for the saturated stage of drying.
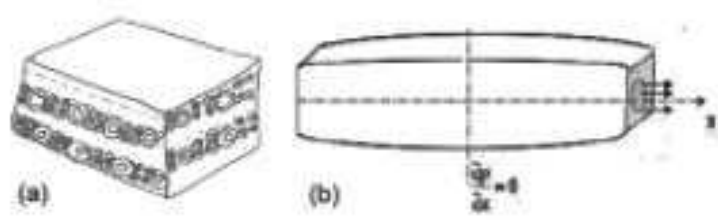

Figure 4. (a) a two-mode system of cylindrical porevessels; (b) individual vessel deformation induced by a pore pressure generated by the external vapor flow.
Eventually air does start to invade into the pore system as shown in Figures 1 and 2, either from boundary or middle of pore system. The event of the air entry inthe former mechanism is interpreted as an external meniscus plunging (Terzaghi 1927) or while the latter consists of a subcutaneous cavitation of water at the boundary. The two are physically undistinguishable phenomena (Brinker \& Scherer 1990). The former suggest a meniscus shrinking until its diameter becomes smaller than the pore entrance. The latter, implying that water suction reaches tensile water strength, depends notoriously on the presence of dissolved air or solid impurities in water. This mechanism yields numerically a criterion based on the Laplace's equation via an equilibrium capillary pressure, $p_{c}^{*}$, which depends on the interface curvature radius, $r$, surface tension, $T_{i}$, and contact angle, $\theta$, as follows

$p_{c}^{*}=\frac{2 T_{s} \cos \theta}{r}, \quad r=a(x, t)$

When capillary pressure rises above this value, $p_{c}^{*}$, the equilibrium is violated and a breakthrough of air is considered to take place. The main point to be stressed is that the change of pore size during drying is substantial, and hence the value of the critical pore size needs to be accordingly adjusted.

We consider a 2D meso-scale assembly for any location along the axial direction of the original soil slab with length $2 \mathrm{~L}$. The air entry mechanism discussed above can be used to simulate possible different sequences of water desaturation in LP or SP, as well as both modes of air entry described in the preceding section. With an example of assembly shown in Figure 4a, the simulation identifies three events of air entry: invasion of air in the LP from its boundary, invasion of air in the SP from its boundary and cavitation of water in the LP-SP intersection due to the pressure in the SP reaching the critical cavitation value in the LP.

The sequence of these three air entry events depends on a number of factors, including evaporation rate, stiffness of LP and SP, configuration of LP and SP, as well as the sizes of LP and SP. A parametric study would exemplify the complex nature of a drying and shrinkage process. The present study utilizes simple parameters discussed in $\mathrm{Hu}$ et al. $(2013 \mathrm{a}, 2013 \mathrm{~b})$ and shows a sequence of desaturation development in different stages.

The subsequent development after the occurrence of air entry is affected by the evaporation at the boundary or in the middle. It should be noted that an approximate formulation for the interface movement is used. Hu et al. (2013a, 2013b) formulated a Stefan problem of moving boundary to account for the motion of the interface. The presented analysis simplified that equation by ignoring 
the small water pressure gradient, and arrives at a formula for the subsequent evolution of the liquid/ gas interface, $s$,

$s=\sqrt{\frac{2 D_{v}\left(c_{e}-c_{0}\right) t}{\rho_{w e}}}$

where $c$ is water vapor concentration, $D_{v}$ is diffusion coefficient of vapor and $\rho_{u}$ is water density.

\subsubsection{Simulation results}

As described in the preceding section, the simulation considers three different processes: Poiseuille flow with an increasing negative pore pressure, invasion of air followed by liquid-vapor interface motion with some pores still satisfying Poiseuille flow and progress of liquid-vapor interface as sole mechanism of water removal at a constant pressure and deformation. The results presented correspond to a meso-scale representative assembly described in Figure 4 , and at $x=L$ in the axial direction.

In the beginning (capillary) stage, large pore and small pore in our 2D model will start to shrink at the same time. Figures 6 and 7 show the evolution of water content and shrinkage, respectively. After reaching at point ' $a$ ' shown in Figure 6 , around $33 \mathrm{hr}$, some of the large pore and small pore at intersection start to desaturate because the pressure developed in the SP reaches the critical cavitation pressure at the LP. As shown in a scenario very similar to what is presented in Figure 2, the air entry scenario, first occurring at middle (intersection of large pore and small pore) is modeled here. Small pore has larger thickness, so its value of "stiffness" as a response to water pressure is higher than that of LP, leading to a larger value
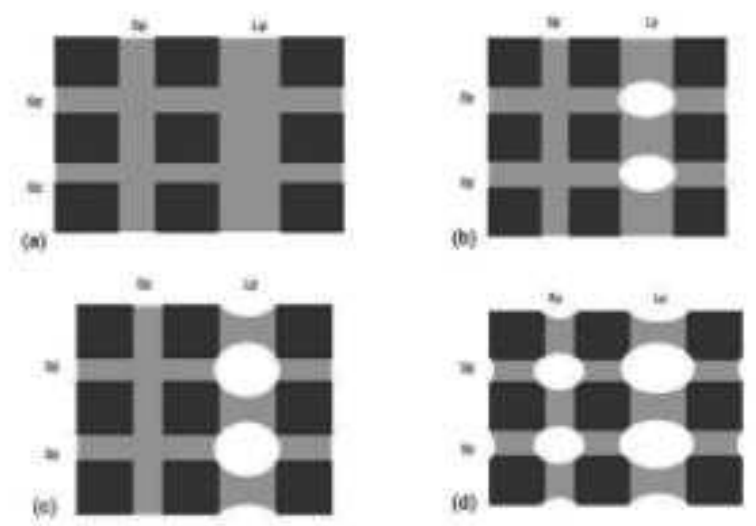

Figure 5. (a) A simple 2D configuration of 3 LP and 9 $\mathrm{SP}$ at initial stage (capillary stage). (b) Air entry at middle LP and SP intersection. It denotes point ' $\mathrm{a}$ ' in the simulated results (start of funicular stage). (c) Air entry into large pores from the boundaries. It refers to point ' $b$ ', (d) Pendular stage of drying. It refers to the region after point ' $c$ ' (end of funicular stage).

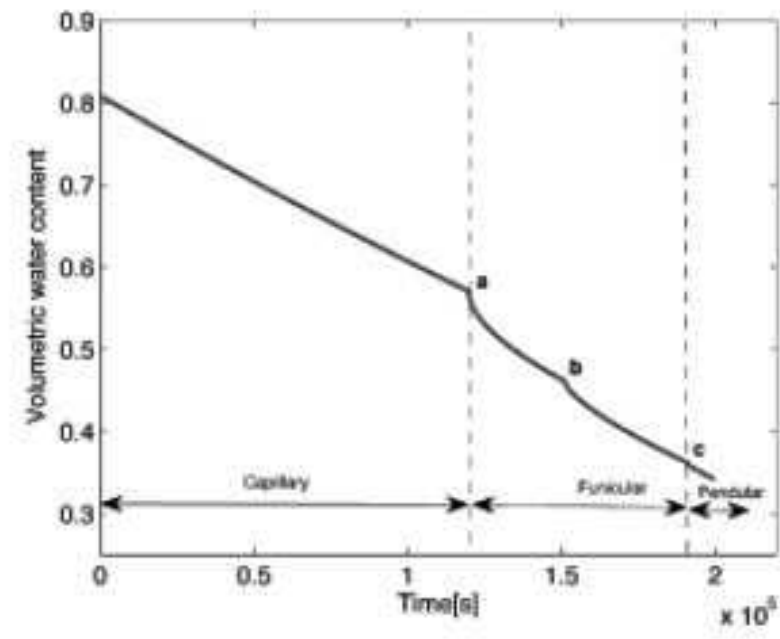

Figure 6. Evolution of volumetric water content simulated.

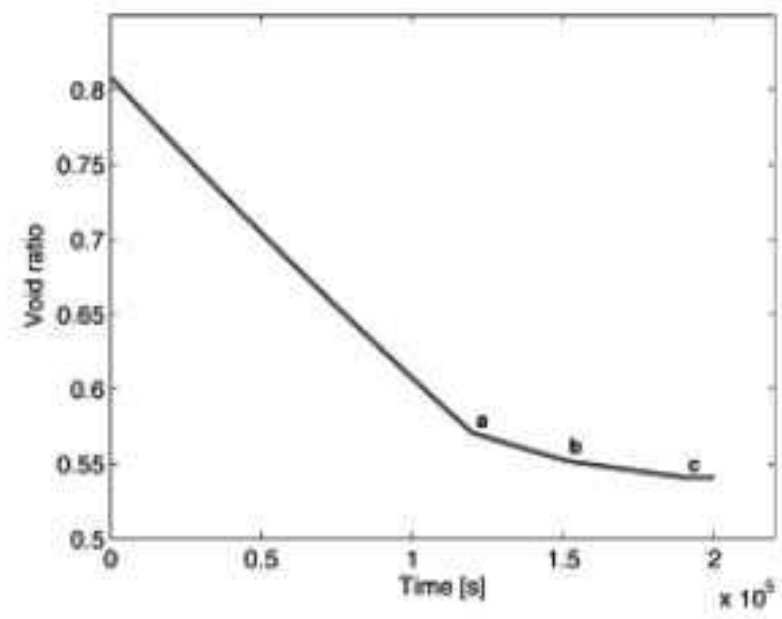

Figure 7. Evolution of void ratio simulated.

of negative pore pressure than that of large pore (if subjected to similar boundary flux condition). It reaches the critical value of large pore around $120,000 \mathrm{sec}$, leading to cavitation at intersection first. This also signifies the beginning of desaturation as shown in Figure 8. This moment can be considered as the beginning of funicular stage for this considered location, as the gas phase is isolated while the liquid phase is continuous (Fig. 5b).

It is interesting to note that the drying rate (loss of volumetric water content) is very high throughout the process. The present model most likely ean be better calibrated for matching the data presented in Figure 3. The key here is that the localized evaporation rate after the air entry was probably overestimated.

The deformation of most large pore and small pore would still continue slowly, and stop for large pore around $42 \mathrm{hr}$ after point ' $b$ ' and for small pore around $53 \mathrm{hr}$ of drying after point ' $\mathrm{c}$ '. Point 


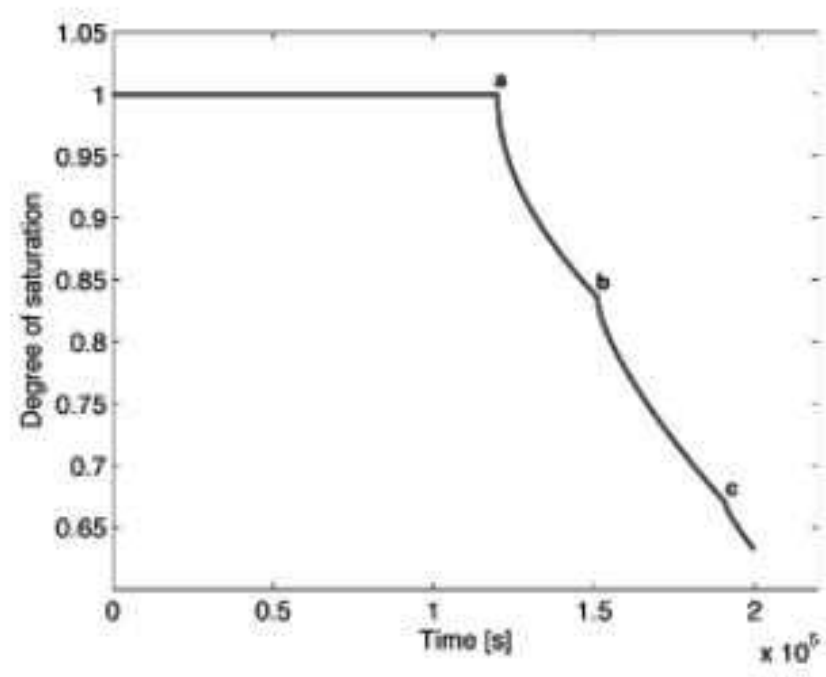

Figure 8. Evolution of degree of saturation simulated.

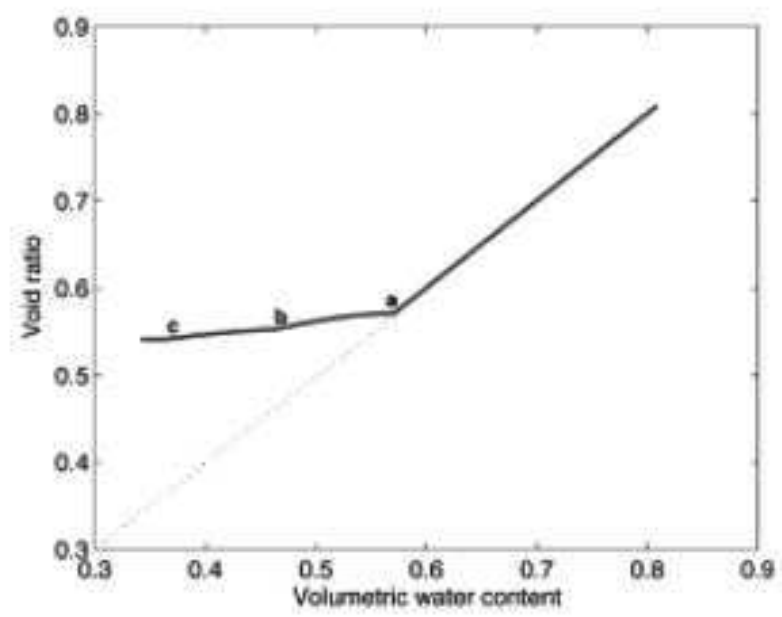

Figure 9. Void ratio versus volumetric water content simulated.

' $b$ ' corresponds to air entry at boundary of large pore around 151,200 sec. However, the boundary here is not referred to as the boundary of the macroscopic soil slab (Fig. 4b), rather, the boundary of the meso-scale representative assembly. It means that if a location rather than the end of slab is considered, the time for ' $b$ ' would come much later, depending on the progress of the interface in the LP. Eventually, small pore is invaded by air at around 191,000 sec. This moment can be considered as the end of funicular stage for this considered location as the liquid phase becomes isolated (Fig, 5d), and possibly the beginning of pendular stage, where the presence of liquid would be limited to the liquid bridges between solid particle.

The initial void ratio and volumetric water content are calibrated against experimental data reported in Peron et al. (2009) and Huet al. (2013c). Figure 9 shows the correlation between void ratio and volumetric water content. It should be pointed out that the characteristic moments in presented model are different from those in the previous ID model, the latter result from homogenization of the entire soil slab while the former have to be considered locally and are distinctly different at different locations.

\section{CONCLUSIONS}

The two-stage development of drying observed at the macro scale are numerically simulated showing the evolution from a constant drying period to a falling rate period. Numerical modeling of drying shrinkage of granular soils is presented based on a conceptual model with the intention to assess the different scenarios for air entry observed in the recent experiments and quantitatively evaluate the implications of these scenarios, leading to the development of different saturation regimes. Only one scenario of air entry is discussed in this paper, air entry at the intersection of large and small pore occur first. However, it might occur first at boundary of large pore as shown in experimental observation at Figure 1, numerically this is possible in the presented model, depending mainly on the stiffness and evaporation rate of small pores. If stiffness and evaporation is much lesser than the value taken during this modeling, air entry at the LP-SP intersection would occur later than at large pore boundary. Obviously the model can be improved if more complicated configurations are considered. However, even sophisticated numerical investigations with Discrete Element suffers from the lack of tools to account for transition of different saturation regimes and thus have to be confined to low degrees of saturation applicable for pendular regime only (c.g. Scholtés et al. 2009). The presented model is able to conceptually show the three regimes: capillary, funicular and pendular during a drying process.

\section{REFERENCES}

Brinker, C. \& G. Scherer (1990). The Physies and Chemistry of Sol-Gel Processing. San Diego: Academic Press.

Chen, W. \& D. Han (1988). Plasticity for Structural Engineers. New York: Springer-Verlag.

Childs E.C. (1969). An Introduction to the Physical Basis of Soil Water Phenomena. London: Wiley.

Fredlund, D. \& H. Rahardjo (1993). Soil Mechanics for Unsaturated Soils. New York: John Wiley \& Sons.

Fung. Y. (1984). Biodynamics: circulation. New York: Springer.

Hill, R. (1950). The Mathematical Theory of Plasticity. Oxford: Oxford University Press. 
Hu, L., H. Peron, T. Hucekel, \& L. Laloui (2013a). Desiccation shrinkage of non-clayey soils: multiphysics mechanisms and a microstructural model. Int. $J$. Numer. Anal. Methods Geomech, 37(12), 1761-1781.

Hu, L. H. Peron, T. Hueckel, \& L. Laloui (2013b). Desiccation shrinkage of non-claycy soils; a numerical study. Int. I. Numer. Anal. Methods Geomech. 37(12), $1782-1800$.

Hu, L., H. Peron, T. Hucckel, \& L. Laloui (2013c). Mechanisms and critical properties in drying shrinkage of soils: experimental and numerical parametric studies. Can. Geotech. J. 50(5), 536-549.

Kodikara, J., S. Barbour, \& D. Fredlund (1999). Changes in clay structure and behaviour due to wetting and drying. Proe of the 8 th Australia-New Zealand Conf. on Geomedhanics, 179-185.

Maeda, N, J.N. Israelachvili, \& M.M. Kohonen (2003). Evaporation and instabilitics of microscopic capillary bridges. Proc. National Academy of Sciences of the USA $100(3), 803.808$.

Mielniczuk, B., T. Hueckel, \& M. El Youssoufi (2013). Micro-scale study of rupture in desiccating granular media. Geo-Congress ASCE GSP 231, $808-817$.

Pellenq, R. B. Coasne, R. Denoyel, \& O. Coussy (2009). Simple phenomenological model for phase transitions in confined geometry. 2. capillary condensation/evaporation in cylindrical mesopores. Largmuir $25(3), 1393-1402$.
Peron, H. T. Hueckel, L. Laloui, \& L.B. Hu (2009). Fundamental of desiccation cracking of fine grained soils: experimental characterization and mechanisms identification. Can. Geotech. J 40(10), 1177-1201.

Peron, H, L. Laloui, \& T. Hueckel (2005). An experimental evidence in desiccation cracking in sandy silt. In Advanced Experimental Unsaturated Soif Medhamies, Taylor \& Francis Group, London, pp. 475-481.

Puyate, Y. \& C. Lawrence (2006). Sherwoods models for the falling-rate period: $A$ missing link at moderate drying intensity. Chem. Eng. Sci. 6l(21), 7177-7183.

Scholtés, L., B. Chareyre, F. Nicot, \& F. Darve (2009). Discrete modelling of capillary mechanisms in multiphase granular media. Computer Modeling in Engineering and Sciences. 52(3), 297-318.

Sherwood, T. (1929). Ithe drying of solids i. Ind. Eng. Chem. 21(1), 12-14.

Taylor, G. (1959). The dynamics of thin shects of fluid. iii. disintegration of fluid sheets. Proc: $R$. Soc. London. Ser. A. 253(1274), 313-1959.

Terzaghi, K. (1927). Concrete roads a problem in foundation engineering. Journal of the Boston Society of Civil Engineers, 14, 265-282.

Urso, M., C. Lawrence, \& M. Adams (1999). Pendular, funicular, and capillary bridges: results for two dimensions. Journal of Colloid and Interface Science. 220(I), $42-56$. 